\title{
Challenges of limited reuse and extended use of N95 filtering facepiece respirators: Lesson learnt from COVID-19 pandemic in Mumbai, India
}

\author{
Patel KP' ${ }^{1}$, Carval TN ${ }^{2}$
}

${ }^{1}$ Consultant Microbiologist, Apoorva Diagnostics \& Healthcare, Bhaktivedanta Hospital \& Research Institute, Thane, ${ }^{2}$ Infection Control Nurse, Bhaktivedanta Hospital \& Research Institute, Thane

\section{ABSTRACT}

Background: Usage of personal protective equipment, which includes N95 filtering face piece respirators (FFRs), was the major preventive measure for healthcare workers to control COVID-19 transmission. However, a global shortage of N95 FFRs was observed worldwide during initial phase of pandemic. Reusing mask was the only strategy and various decontamination methods were suggested. The main objective of the study was to evaluate the compliance rate of limited reuse and extended use policy implemented in hospital.

Methods: Limited reuse and extended use policy of N95 FFRs was implemented for one month in lower risk areas. Compliance to policy was checked by questionnaires asked to 100 healthcare workers verbally and challenges faced by them were noted.

Results: It was observed that overall compliance to policy was $80 \%$. Major reasons of non-compliance were suffocation, followed by smell from mask and loosened strips.

Conclusion: Limited reuse and extended use method was successfully used as a bridge until sufficient N95 FFRs were not available although effective decontamination method must be established in hospitals to prepare ourselves for the future pandemics.

Key words: Compliance, COVID-19, Extended use, Healthcare workers, Limited reuse, N95 FFRs

\section{Introduction}

$A^{n}$ novel coronavirus, severe acute respiratory syndrome coronavirus 2 (SARS-CoV-2), causing coronavirus disease 2019 (COVID-19), emerged in Wuhan City, China posed a global health threat, causing an ongoing pandemic in many countries and created a major public health issue. ${ }^{1}$ The most important measures recommended were suspected source control, use of personal protective equipment, rapid

\section{DOI: https://doi.org/10.3126/ijosh.v11i2.37253}

Conflicts of interest: None

Supporting agencies: None

Date of submission: 24.05 .2021

Date of acceptance: 29.06.2021

Date of publication: 30.06 .2021

Corresponding Author
Dr. Kinjal P Patel
Apoorva Diagnostics \& Healthcare,
Bhaktivedanta Hospital \& Research Institute, Mira Road,
Thane
Tel: +919724310931
E-mail: kinjal.1527@gmail.com
ORCID ID: https://orcid.org/0000-0001-6525-3621

diagnosis, physical distancing, isolation, investigation and follow-up of close contacts. As it was evident from the continuously increased number of confirmed cases globally, that COVID-19 had severe pathogenesis as well as higher transmission competence. However, the major concern for healthcare settings was numerous reported cases of nosocomial transmission in hospitals. ${ }^{2}$ The key factor for prevention and control was use of personal protective equipment and one of the most important personal protective equipment to prevent transmission and community spread was facemasks, which were in extreme short supply during the peak of pandemic.

Facemasks are typically engineered and specified for single use only. ${ }^{3}$ But to meet the increased demand in times of crisis, rapid and effective decontamination methods were evaluated. ${ }^{4}$ Centers for disease control and prevention provided a guideline for various

(c) (i) (8)
This journal is licensed under a Creative Commons Attribution-
Non Commercial 4.0 International License.


decontamination methods. All different methods were evaluated, their effectiveness against various microbes were tested, treatment levels assessed and the antimicrobial efficacy tested. Vaporous hydrogen peroxide, ultraviolet germicidal irradiation, and moist heat were found to be the most promising decontamination methods that will not appear to break down filtration or compromise the FFRs. However, such methods can only be used for limited number of cycles. Steam treatment and liquid hydrogen peroxide too were effective methods with limitations. ${ }^{5}$ Ethylene oxide was not recommended for FFRs decontamination, as it is carcinogenic, teratogenic, and harmful to the wearer even at very low levels. ${ }^{6}$

However, some other cost effective methods evaluated like autoclave, $160^{\circ} \mathrm{C}$ dry heat, $70 \%$ isopropyl alcohol, microwave irradiation but they caused substantial filter degradation of FFRs, and even particle penetration levels did not meet the levels that NIOSH (The National Institute for Occupational Safety and Health, USA) would allow for approval. ${ }^{7,8}$ Meanwhile, reusing disposable FFRs had been suggested as a crisis capacity strategy to preserve available supplies for healthcare workers during a pandemic. ${ }^{5}$ Since, procurement of new equipment for decontamination was difficult, limited reuse and extended use of N95 FFRs was implemented for healthcare workers in hospital. Due to scarce knowledge about reuse of N95 FFRs and apprehension amongst healthcare workers, it was important to monitor the compliance rate. Hence, main objective of the study was to assess the compliance of healthcare workers to limited reuse and extended use policy and to evaluate the reasons of non-compliance.

\section{Methods}

A healthcare facility which is a 250 -bedded tertiarycare hospital, located in North Mumbai, India with approximately 20000 annual admissions in a catchment area of 20 lakh persons was in the midst of COVID 19 outbreak. In the month of April 2020, due to sudden surge in demand of N95 FFRs, hospital infection control team decided to implement limited reuse and extended use of N95 FFRs because hospital were admitting COVID-19 patients. Based on clinical judgment and the availability of institutional resources, simplest strategy of masks rotation was implemented for all healthcare workers excluding those of high-risk areas. Seven masks were given to healthcare workers with seven paper bags. Instructions were given to use N95 mask only in hospital premises and should only be worn and used by a single wearer. Cloth masks or triple layer surgical masks to be use in community. Step wise protocol was given that includes following points:

1. Provision of seven masks with seven paper bags labeled days Monday to Sunday.

2. Each N-95 mask in separate paper bag and marked with user's name and day.

3. On day 1(Monday), mask No.1 to be used on joining duty. After finishing the duty, mask should be kept in paper bag No.1 and allowed it to dry out for 6 days.

5. Same protocol to be followed for all other days until Sunday.

7. Repetition of mask usage from next week again and repeat use until all seven masks have been used 5 times.

8. Cycle should be repeated 5 times. Discard all seven masks when used up in 35 days. Issuing of new masks after 35 days.

9. Instructions given regarding procedure for donning and doffing of masks.

10. Extended use was promoted as per the work hour pattern, which was 8 hours for all healthcare workers.

11. Instructions given to discard FFRs when contaminated with blood, respiratory or nasal secretions, or other bodily fluids from patients or following close contact with any patient coinfected with an infectious disease requiring contact precautions.

Total 150 employees working in non-clinical areas like pharmacy, spiritual care department, accounts, biomedical engineering, administration, maintenance department were instructed accordingly and training for limited reuse and extended use of masks given. After one month of implementation, compliance for limited reuse and extended use of N95 FFRs was checked in the month of May 2020. Infection control nurse interacted for feedback with total 100 health care workers who were following policy and on duty during assessment day. Questions were asked to healthcare workers verbally and challenges faced by them were noted. After two months of ongoing pandemic, in view of increased production capacity and increased availability of N95 FFRs, limited reuse and extended use policy was stopped. Ethical approval taken from Institutional ethics committee of Bhaktivedanta hospital and research institute for data analyses. 


\section{Results}

It was observed that overall compliance of healthcare workers to limited reuse and extended use of N95 FFRs was $80 \%$. [Figure 1]

Major reasons of non-compliance were suffocation (29\%) followed by smell from mask (13\%) and loosening of strips $(9 \%)$. Other reasons were refreshment $(8 \%)$, itching $(8 \%)$, soiling of masks $(7 \%)$, only wearing when patients were nearby $(6 \%)$, fungal growth inside mask (6\%) and dehydration issue (5\%). Less common reasons were training not given, not wearing during academic hours and departmental change while working. [Figure 2] Other aspects observed were place of storing the masks. It was noted that all healthcare workers were following protocol of keeping one mask in one bag and storing those bags at dry place.

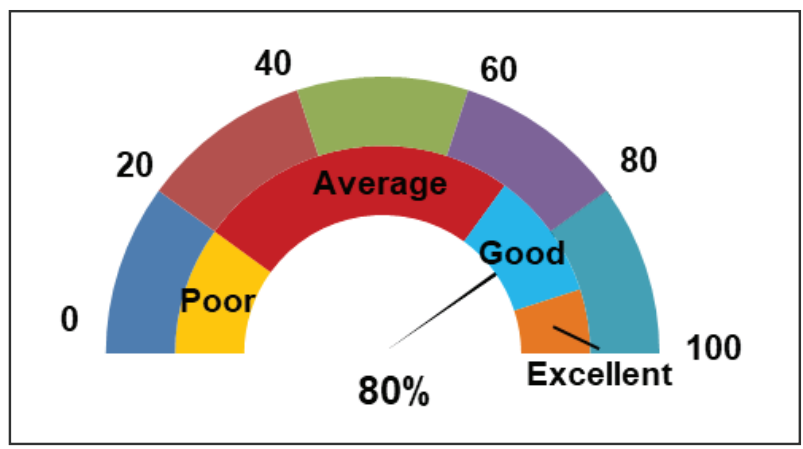

Figure 1: Compliance to extended use and limited reuse policy of N95 FFRs

\section{Discussion}

Due to shortage of N95 FFRs worldwide, Centers for disease control and prevention gave guidelines for limited reuse and extended use of FFRs in healthcare settings. ${ }^{9}$ As per the definition, extended use refers to the practice of wearing same N95 respirator for repeated close contact encounters with several patients, without removing the respirator between patient encounters. Reuse refers to the practice of using the same N95 respirator for multiple encounters but removing it after each encounter. The respirator is stored in between exposures so that can be used again for the next encounter with a patient. Although reuse of N95 respirator was recommended, but no method was available to check the durability of N95 FFRs. Hence, it is essential to limit the number of times the same N95 FFR is reused. Thus, limited reuse has been recommended and used widely during previous outbreaks as an option for conserving respirators.

Van Doremalen et al. provided evidence of virus viability and noted that virus can remain infectious in aerosols for hours and on surfaces up to days depending on the inoculum shed. Study noted that COVID-19 virus survived 4 hours on copper, 24 hours on cardboard and up to 72 hours on plastic and stainless steel. ${ }^{10}$ Another study done by Chin et al found that the COVID-19 virus remained viable up to 1 day on cloth and wood, up to 2 days on glass, 4 days on stainless steel and plastic and up to 7 days on the outer layer of a medical mask. ${ }^{11}$ Juang et al recommended that N95 masks can be rotated, using 3-4 masks numbered outside as one to four in numerical order can be used every 3-4

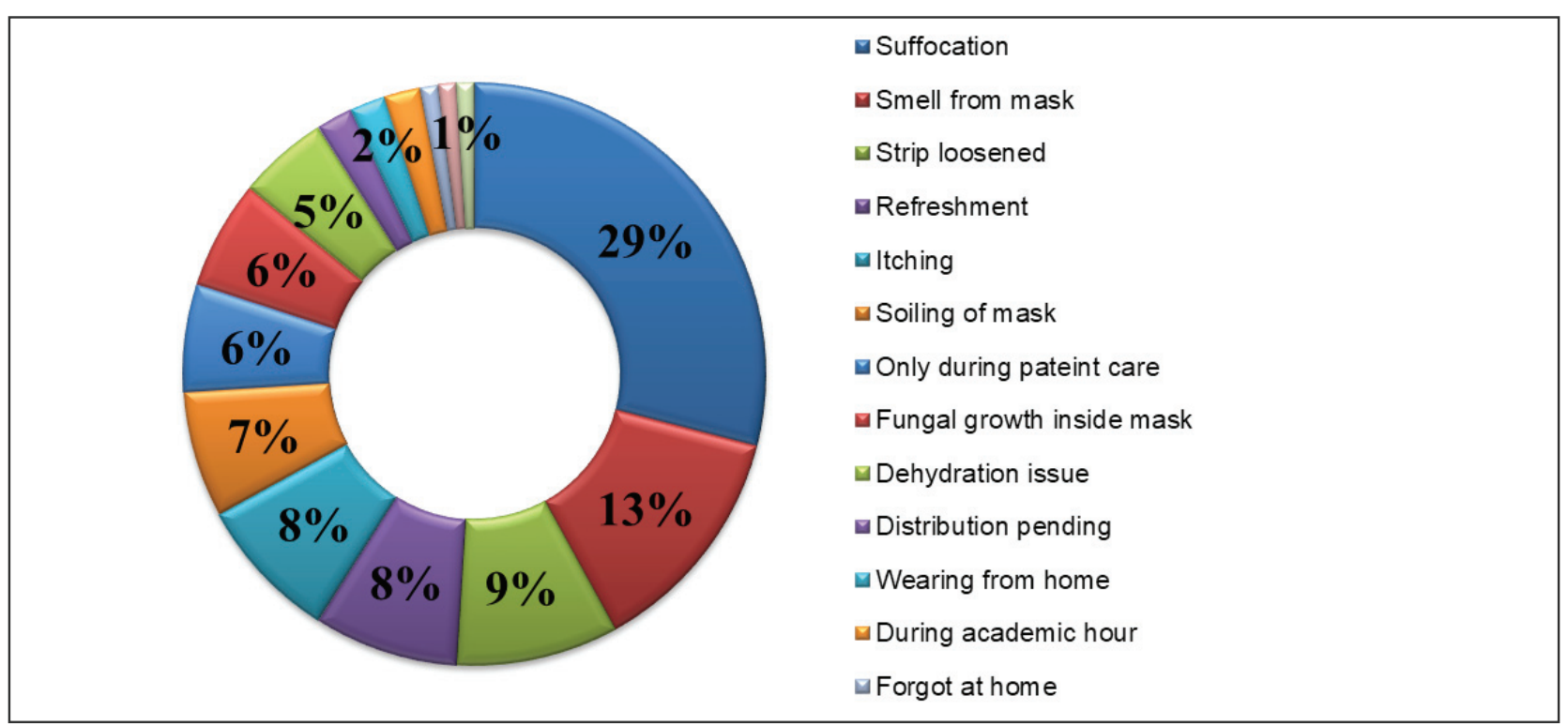

Figure 2: Reasons for non- compliance to extended and limited reuse policy of FFR 
days and noted that if masks were rotated and kept at room temperature $\left(21-23^{\circ} \mathrm{C}\right)$ with $40 \%$ humidity, there was no change in the mask's properties. ${ }^{12}$ Hence, from above studies, it was concluded that clinically significant amount of viable virus on masks were unlikely after few days.

In present study, hospital administration decided to give N95 FFRs to all healthcare workers despite being working at non-clinical areas to lessen apprehension. All critical care areas were excluded as aerosolgenerating procedures are being carried out in critical care units. Therefore, risk of splashes and droplets exposure was more. If viral load is high in droplets, they will settle on masks and reusing the mask would be a greater risk to healthcare workers.

The compliance of limited reuse and extended use of N95 FFRs was $80 \%$. Considering many COVID-19 patients being asymptomatic, all healthcare workers strictly followed the policy and gave satisfactory feedback. The major reasons of non-compliance were suffocation $(29 \%)$ followed by smell from mask $(13 \%)$ and loosening of strips $(9 \%)$ which were practically challenging. Other reasons were removal of mask for refreshment $(8 \%)$, itching $(8 \%)$, soiling of masks $(7 \%)$, and only wearing when patients were nearby $(6 \%)$, fungal growth inside mask (6\%) and dehydration issue $(5 \%)$.To raise the compliance amongst healthcare workers, N95 FFRs brand was changed and new masks were given. After two months, considering the increased production of N95 FFRs, limited reuse and extended use policy was stopped.

\section{References}

1. Rodriguez-Morales AJ, Bonilla-Aldana DK, BalbinRamon GJ, Rabaan AA, Sah R, Paniz-Mondolfi A et al. History is repeating itself: Probable zoonotic spillover as the cause of the 2019 novel Coronavirus Epidemic. Infez Med. 2020; 28(1):3-5.

2. McMichael TM, Currie DW, Clark S, Pogosjans S, Kay M, Schwartz NG, et al. Epidemiology of Covid-19 in a Long-Term Care Facility in King County, Washington. N Engl J Med. 2020; 382(21):2005-11.

3. Mackenzie D. Limited reuse of N95 Masks. Engineering (Beijing). 2020; 6(6):593-96.

4. Decontamination and Limited reuse of Filtering Facepiece Respirators.2020. Available from: https:// www.cdc.gov/coronavirus/2019-ncov/hcp/ppe strategy/decontamination-limited reuse-respirators. html.
Infection control practices are of paramount importance in any healthcare settings. Inappropriate infection prevention measures and limited awareness about all transmission dynamics of emerging pathogens created a biggest challenge and threat to healthcare workers. Some issues for example removing masks because of dehydration and refreshment activities were difficult to resolve but maintaining uniformity in the institutional guidelines and monitoring of implementation of the policy should be prioritized in pandemic crisis.

COVID-19 caused millions of death worldwide and its long-term health effects will contribute to have devastating consequences. There is a need for enforcement of existing laws on infection prevention in all healthcare settings and implementation of systematic strategies.

\section{Conclusion}

Limited reuse and extended use of N95 FFRs can be used as a short-term measure. To improve the compliance, the shorter rotation cycles and good quality N95 FFRs must be used. Besides all healthcare workers needed to be trained properly for using N95 FFRs properly. Although the major hurdle for resource-limited countries are institutional framework and affordability, effective and budget friendly decontamination methods are needed for reuse of masks without degrading the quality of FFRs. The COVID-19 pandemic might be the worst experience for all but we need to learn and act if we have to be ready for the next contagion.

5. Centers for Disease Control and Prevention website. Decontamination and limited reuse of filtering facepiece respirators. Available at: https://www. cdc.gov/coronavirus/2019-ncov/hcp/ppe-strategy/ decontamination-limited reuse-respirators.html. Accessed, October 19, 2020.

6. Rutala, WA, Weber DJ. Guideline for disinfection and sterilization in healthcare facilities, 2008. Available from: https://www.cdc.gov/infectioncontrol/guidelines/ disinfection/.

7. Viscusi DJ, Bergman MS, Eimer BC, Shafferet $\mathrm{RE}$. Evaluation of five decontamination methods for filtering facepiece respirators. Annals of occupational hygiene 2009; 53(8):815-27.

8. Viscusi DJ, King W, Shaffer RE. Effect of decontamination on the filtration efficiency of two filtering facepiece respirator models. Journal of the 
International Society for Respiratory Protection 2007; 24:93-107.

9. National Institute for Occupational Safety and Health. Recommended guidance for extended use and limited reuse of N95 filtering facepiece respirators in healthcare settings. Centers for Disease Control and Prevention website. Published March 2020.

10. Van Doremalen N, Bushmaker T, Morris DH, Holbrook MG, Gamble A, Williamson BN, et al. Aerosol and surface stability of sars-cov-2 as compared with SARS-CoV-1. N Engl J Med 2020; 382:1564-7.

11. Chin AWH, Chu JTS, Perera MRA, Hui KPY, Yen $\mathrm{HL}$, Chan MCW, et al. Stability of SARS-CoV-2 in different environmental conditions. Lancet Microbe. 2020;1(1):e10.

12. Juang PSC, Tsai P. N95 Respirator Cleaning and Limited reuse Methods Proposed by the Inventor of the N95 Mask Material. J Emerg Med. 2020; 58(5):817-20. 